\title{
Comparison of LULU and Median Filter for Image Denoising
}

\author{
R. Rahmat, A. S. Malik, and N. Kamel
}

\begin{abstract}
This paper presents a comparison between LULU and Median filters for impulse noise in images. Noise removal from images is always a challenging area of research. Different methods are being used for different image noises such as Wiener filter for Gaussian noise, Frost filter for speckle noise and median filter for impulse noise. LULU filters are widely being used for impulse noise too. LULU filters are nonlinear rank selector operators which are computationally more competent and the performance of the operator is straightforward to describe. The comparison between Median and LULU filters, in this paper, is performed using two different image quality metrics which are RMSE and PSNR.
\end{abstract}

Index Terms - LULU filters, impulse noise, RMSE, PSNR.

\section{INTRODUCTION}

In the field of image processing, there are so many issues and complexities for the recovery of original data from the noise corrupted data. In general, image noise is the variation of color or brightness intensity which can be caused by different sources. In general, image sensors degrade the quality of the images. Faulty devices, troubles with the data collection procedure, and interfering natural cause can all corrupt the data. Moreover, noise can be caused by compression and transmission errors. Thus, noise removal is required and is the first step before images are analyzed. It is necessary to apply an efficient noise reduction method to compensate for such data corruption [1], [2]. Even though there are so many different methodologies in this field for noise removal, image denoising is still a big issue. Some noise filters cause blurring of the images, because in reality the nature of the noise is unknown or only known to some extent.

Impulse noise results due to the malfunctioning of the image sensors and the transmission channels. It can be identified by black and white points in the image. So far, there are so many different algorithms that had been proposed to filter this type of noise such as median, switching median, weighted median, adaptive median, rank conditioned median, separable median and contra harmonic mean filter. There are different names for impulse noise which are salt and pepper as well as shot noise.

In this work, we address impulse noise. Generally, median filter is used for reducing impulse noise. We compared four different LULU filters with median filter. This comparison

Manuscript received April 20, 2013; revised June 28, 2013.

R. Rahmat is with the Institute of Sensors, Signals and Systems, Heriot-Watt University, UK (e-mail: roushanakrahmat@gmail.com).

A. S. Malik is with the Mission Oriented Research Biomedical Technology at Universiti Teknologi Petronas.

$\mathrm{N}$. Kamel is with the PETRONAS University of Technology. has been done through two different image quality measures which are; Root Mean Square Error (RMSE) and Peak Signal to Noise Ratio (PSNR) techniques. Two test images are used for experiment and they are shown in Fig. 1.

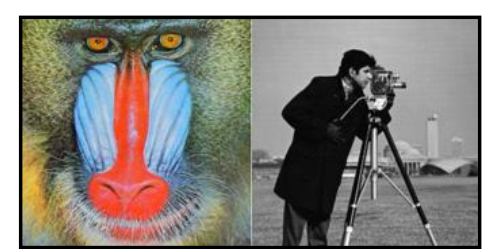

Fig. 1. Test images, from left to right: baboon and cameraman images

\section{MEDiAn FILTER}

Median filter for images, as its name represents, substitutes the pixel value with the neighbor values of that pixel. Median filter is one of the most popular order statistics, nonlinear filters. It is extensively applied in digital image processing due to its property of protecting edges as well as at the same time eliminating noise but it causes the image to blur. But still Median filter causes significantly a smaller amount of blurring than linear smooth filters of the same size. In general, all Median filters produce reasonable results for both bipolar and unipolar impulse noise. They are nonlinear, non-idempotent smoother which is computationally less efficient than LULU.

\section{A. $1 D$ Median}

In one dimensional arrays, median filter is given by:

$$
M_{n}(x)_{i}=\operatorname{median}\left\{x_{i-n}, \ldots, x_{i}, \ldots, x_{i+n}\right\}
$$

The Fig. 2 illustrates the median operator in filtering a sequence. In this figure, the top one is the original signal and the bottom one shows the smoothed signals after applying median filter.

\section{B. 2D Median}

$$
\begin{aligned}
& M\left(f\left(x_{i j}\right)\right) \\
& =\operatorname{median}\left\{f\left(x_{i, j-1}\right), f\left(x_{i, j+1}\right), f\left(x_{i, j}\right), f\left(x_{i-1, j}\right), f\left(x_{i+1, j}\right)\right\}
\end{aligned}
$$

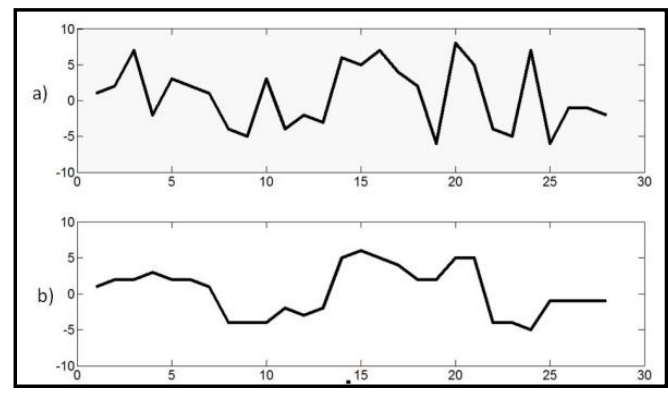

Fig. 2. a) Original signal, and b) Result of median filter on the signal 


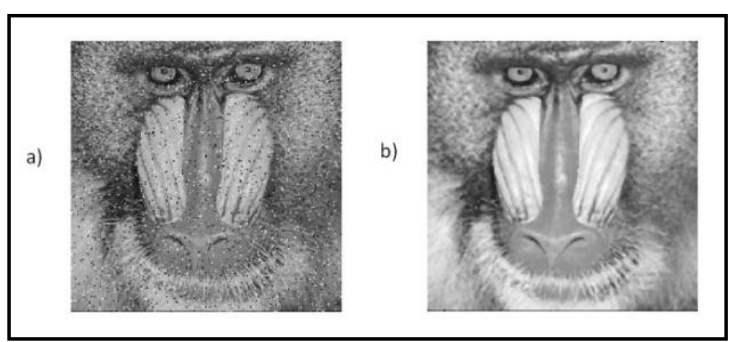

Fig. 3. a) Noisy image with impulse noise, and b) Filtered image with median filter

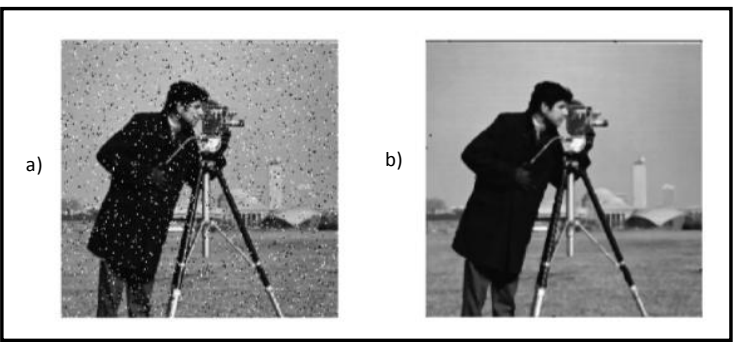

Fig. 4. a) Noisy image with impulse noise, and b) Filtered image with median filter

For images, i.e., two dimensional arrays, median filter is given in (2), where $f$ refers to the image and $\left(x_{i j}\right)$ is the pixel. The effect of median filter after removing the impulse noise of baboon and cameraman images are shown in Fig. 3 and Fig. 4 respectively.

\section{LULU OPERATORS}

Rohwer and Toerien in late 1980s introduced a novel innovative non-linear smoother, named LULU smoothers, based on extreme order statistics [3]. Initially LULU operators were evaluated for removal of impulse noise of sequences in [4]. LULU filters are computationally suitable and theoretically simpler contrast to the median filters which are fenally considered to be the "basic" smoothers [5], [6].

LULU filters are being widely used in statistics as shown in [7] and recently it is implemented in object extraction of images by applying DPT method in [6], [8] and [9]. They are local, non-linear filters used for the elimination of salt and pepper noise. LULU operators combined the two basic operators L (low) und U (upper) with special arrangement in different filters.

LULU operators can be used for signals in one dimensional arrays as well as images in two dimensional arrays for smoothing or filtering the image and also for object detection and extraction (by using DPT) with composition of different $\mathrm{L}$ and $\mathrm{U}$ operators.

\section{A. $1 D-L U L U$}

LULU filters the signal by performing a simple assessment method to remove the local peaks and valleys. For a particular bi-infinite sequence $\xi=\left(\xi_{i}\right)_{\mathrm{i} \ell Z}$, the one dimensional LULU operators are defined as follows [9]:

$$
\begin{gathered}
\left(L_{n} \xi\right)_{i}=\max \left\{\min \left\{\xi_{i-n}, \ldots, \xi_{i}\right\}, \min \left\{\xi_{i}, \ldots, \xi_{i+n}\right\}\right\}, i \in Z \\
\left(U_{n} \xi\right)_{i}=\min \left\{\max \left\{\xi_{i-n}, \ldots, \xi_{i}\right\}, \max \left\{\xi_{i}, \ldots, \xi_{i+n}\right\}\right\}, i \in Z
\end{gathered}
$$

Fig. 5 illustrates the results of $L$ and $U$ operators in filtering the sequence. In this figure, the top one is the original signal while the middle and the bottom one show the smoothed signals after applying $L$ and $U$ operators respectively.

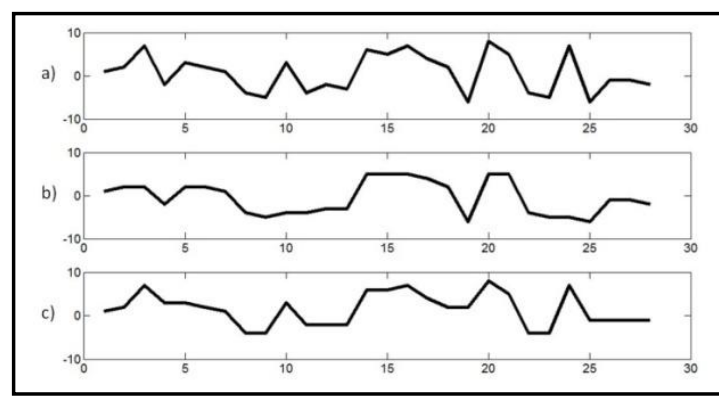

Fig. 5. a) Original signal, b) Result of $L$ smoother on the signal, and c) Result of $\mathrm{U}$ smoother on the signal

\section{B. $2 D-L U L U$}

For applying LULU on images, we need to compare any single pixel with all the neighbors in the selected window. We can classify the neighborhood of a pixel in different ways such as the ones shown in the Fig. 6.

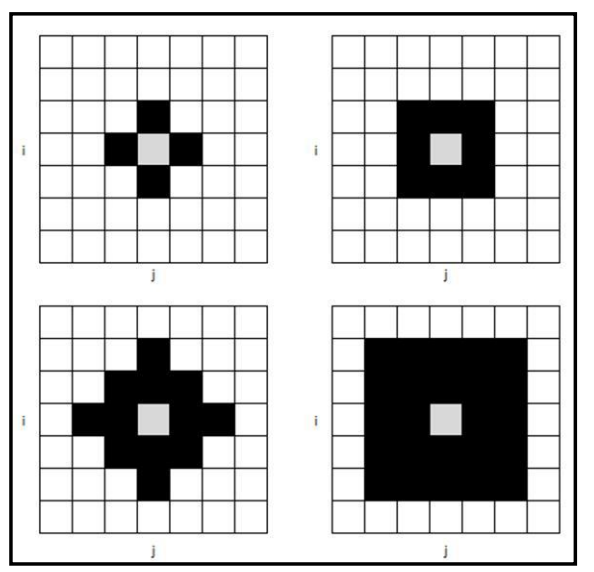

Fig. 6. Four different neighboring regions of pixel $(i, j)$

For more explanation about $2 \mathrm{D}$ processing using LULU operators, an example is shown as follows. We segment the neighbors of the pixel $I(i, j)$ to four different parts as shown in (5) to (8) and Fig. 7.

$$
\begin{aligned}
& I_{1}=[I(i-1, j-1), I(i-1, j), I(i, j), I(i, j-1)] \\
& I_{2}=[I(i-1, j+1), I(i-1, j), I(i, j), I(i, j+1)] \\
& I_{3}=[I(i, j-1), I(i, j), I(i+1, j-1), I(i+1, j)] \\
& I_{4}=[I(i, j+1), I(i, j), I(i+1, j), I(i+1, j+1)]
\end{aligned}
$$

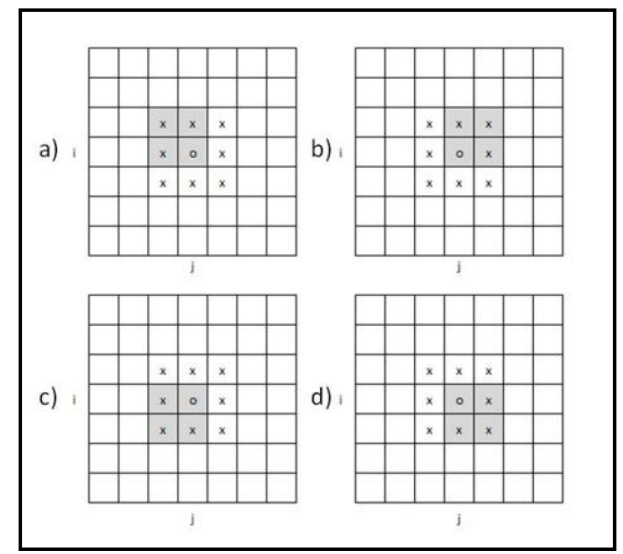

Fig. 7. Illustration of neighbors for (3) to (6), a) I1, b) I2, c) I3, and d) I4 
Then we applied the $\mathrm{L}$ and $\mathrm{U}$ operators as follow:

$$
\begin{gathered}
L(i, j)=\max (\min (I I), \min (I 2), \min (I 3), \min (I 4)) \\
U(i, j)=\min (\max (I I), \max (I 2), \max (I 3), \max (I 4))
\end{gathered}
$$

The order of applying L, U operators depends on the design of filter, for example, LU filter perform opening between $\mathrm{L}$ and $\mathrm{U}$ operators or in a simple way, we can say that after applying $L$ on the image, we apply $U$ on the result of $\mathrm{L}$ operator and the final result is LU filter result. For detailed discussion of properties of LULU operators, as well as their proofs, check references [5], [6].

The Fig. 8 a) illustrates a randomly generated binary image and Fig. 8 b) and Fig. 8 c) show the filtered images after applying $\mathrm{L}$ and $\mathrm{U}$ filters separately on the binary image. In this example, the binary image has balanced number of black and white parts. After applying L smoother on the image, we can see that the black parts are greater than before. It can be explained based on (9). L operator gets the most out of the minimums of the neighborhood which actually removes the lower peaks (this is the reason that Fig. 8 b) has more black marks than the original image). $\mathrm{U}$ smoother is opposite to $\mathrm{L}$ smoothers. We see that Fig. $8 \mathrm{c}$ ) is whiter in compare with the original image. Other examples are given in Fig. 9, to illustrate the concept of $\mathrm{L}$ and $\mathrm{U}$ filters on corrupted images with impulse noise.

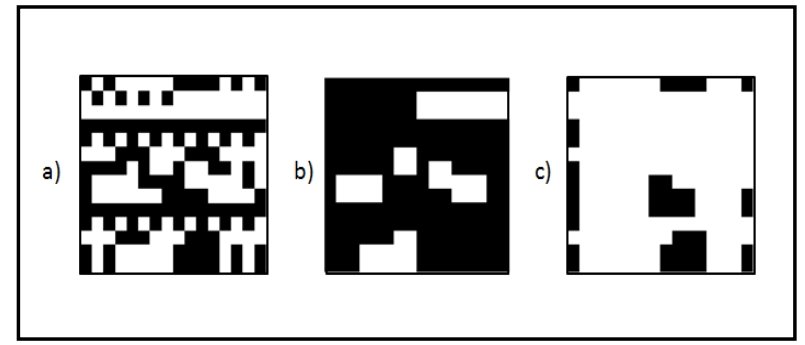

Fig. 8. a) Original binary image, b) Result of L smoother on the image, and c) Result of $\mathrm{U}$ smoother on the image

a)
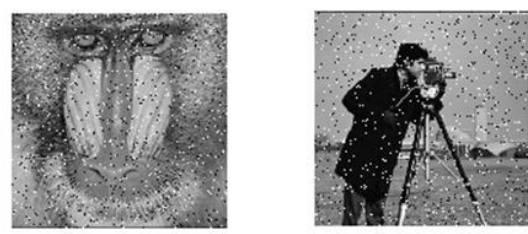

b)
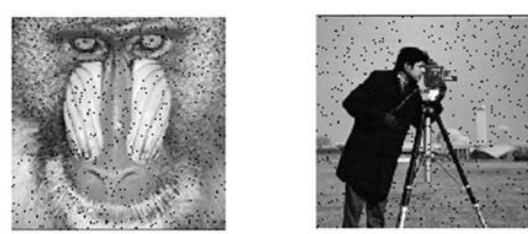

c)
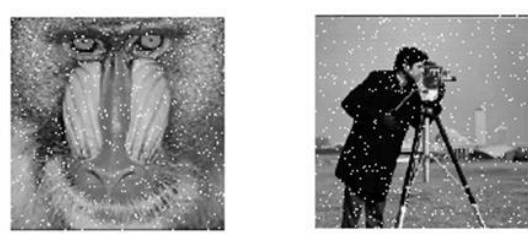

Fig. 9. a) Corrupted image with impulse noise, b) Result of L smoother on the image, and c) Result of $U$ smoother on the image

\section{IMPULSE NOISE REMOVAL}

LULU filters are compared with the median filter with the noise density of impulse noise varying from 0.01 to 1.00 with 0.05 step size increment each time. Some of the normalized comparison results are illustrated and compared in Fig. 10 and Fig. 11.

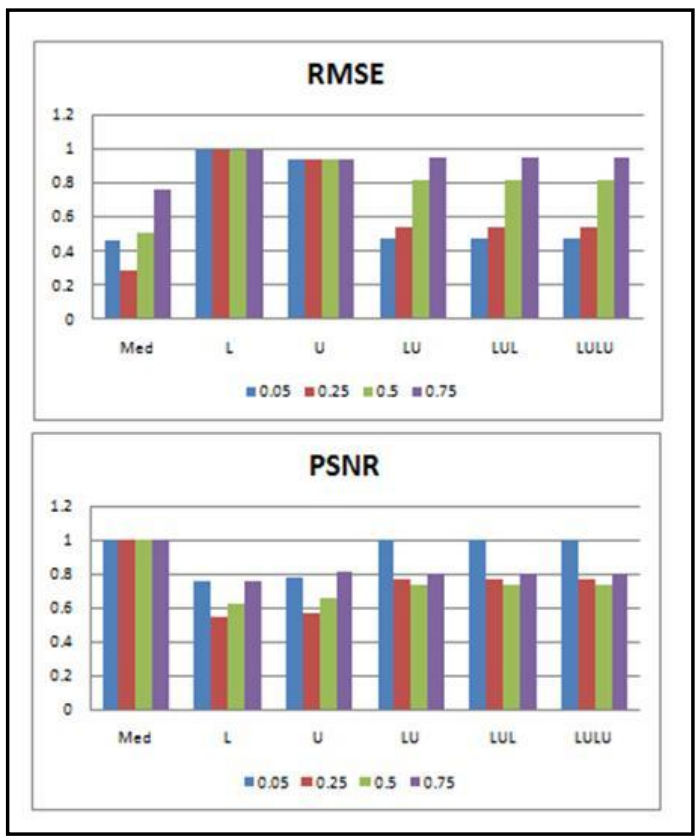

Fig. 10. RMSE and PSNR results in comparison of LULU and median filter for baboon image

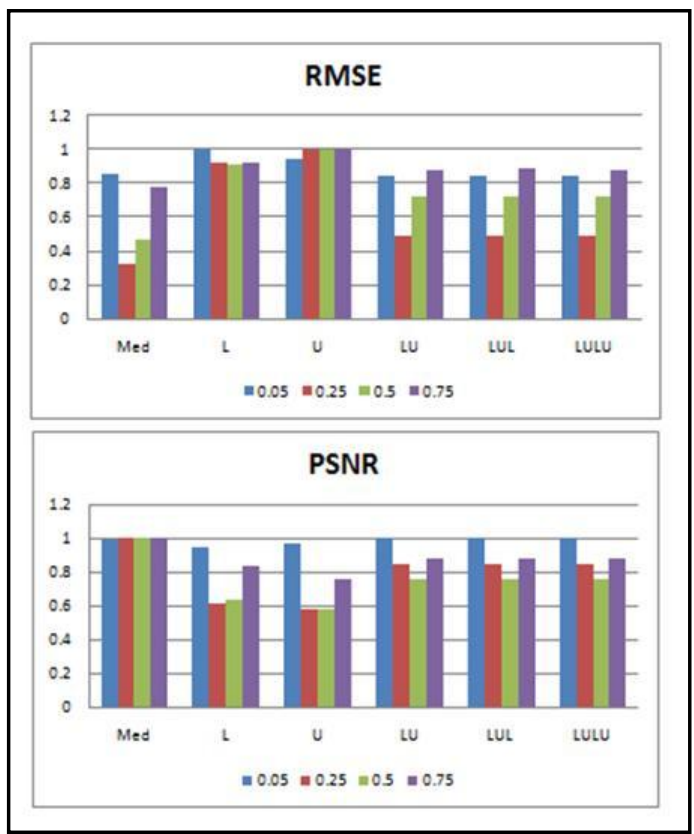

Fig. 11. RMSE and PSNR results in comparison of LULU and median filter for cameraman image

\section{CONCLUSION}

In this work we have compared LULU filters with Median filter for image denoising. The input captured images were assumed to be corrupted by impulse noise. Two different image quality measures, RMSE and PSNR are used to compare the result of LULU and median filters. The obtained result shows that LULU filters are not always performing 
better than other filter. Generally, the result of median and LULU are comparable for different impulse noise densities. In future, we plan to test LULU filters for various applications like 3D recovery [10], microscopic applications [11], communication [12], medical imaging [13], [14], etc.

\section{ACKNOWLEDGMENT}

This work is supported by the E-Science grant funded by the Ministry of Science, Technology and Innovation (MOSTI), Government of Malaysia (No: 01-02-02-SF0064).

\section{REFERENCES}

[1] M. Motwani, M. Gadiya, and R. Motwani, "Survey of image denoising techniques," in Proc. GSPx, Santa Clara, CA., pp. 27-30, 2004.

[2] S. Shim, A. S. Malik, and T. Choi, "Pre-processing for noise reduction in depth estimation," in proc. 2nd International Conference on Digital Image Processing, SPIE vol. 7546, pp. 754625-2 7, 26-28 February, 2010.

[3] W. J. Conradie, T. D. Wet, and M. Jankowitz, "Exact and asymptotic distributions of LULU smoothers," Journal of Computational and Applied Mathematics, vol. 186, no. 1, pp. 253 - 267, February 2006.

[4] T. de Wet and W. Conradie, "Smoothing sequences of data by extreme selectors," in Proc. ICOTS-7, pp. 1-5, 2006.

[5] C. H. Rohwer and D. P. Laurie, "The discrete pulse transform," SIAM J. Math. Anal, vol. 38, no. 3, pp. 1-28, 2007.

[6] J. P. D. Toit, "The discrete pulse transform and applications," Masters Thesis, University of Stellenbosch, 2007.

[7] W. J. Conradie and T. de Wet, "LULU smoothers and their statistical properties," Technical Report 03/01, Department of Statistics and Actuarial Science, University of Stellenbosch, South Africa, 2005, pp. $1-37$.

[8] B. Anguelov, "Discrete pulse transform of images: Algorithm and applications," in Proc. Int. Conf. Pattern Recognit., Tampa, FL, pp. 8-11, 2008.

[9] R. Anguelov and I. Fabris-rotelli, "LULU operators and discrete pulse transform for multidimensional arrays," Image (Rochester, N.Y.), vol. 19, no. 11, pp. 3012-3023, 2010.

[10] A. Malik and T. Choi, "Comparison of polymers: a new application of shape from focus," IEEE Transactions on Systems, Man, and Cybernetics--Part C: Applications and Reviews, vol. 39, no. 2, pp. 246-250, 2009

[11] S. Shim, A. Malik, and T. Choi, "Accurate shape from focus based on focus adjustment in optical microscopy," Microscopy Research and Technique, vol. 72, no. 5, pp. 362-370, 2009.

[12] J. Khodjaev, Y. Park, and A. Malik, "Survey of NLOS identification and error mitigation problems in UWB-based positioning algorithms for dense environments," Springer Annals of Telecommunications, vol. 65 , no. 5, pp. 301-311, 2010.

[13] A. Malik, "Simulation-based analysis of the resolution and SNR properties of partial k-space EPI," Concepts in Magnetic resonance Part B Magnetic Resonance Engineering, vol. 35B, no. 4, pp. 232-237, 2009.

[14] A. Malik and T. Choi, "Differentiating honeycombed images from normal HRCT lung images," IEICE Transactions on Information \& Systems, vol. E92-D, no. 5, pp. 1218-1221, 2009.

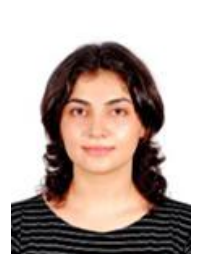

Roushanak Rahmat received the BS and MS degrees in Electrical and Electronics engineering from Universiti Teknologi PETRONAS, Malaysia, 2009 and 2012, respectively. She is currently a PhD student at Institute of Sensors, Signals and Systems, Heriot-Watt University, UK. Her research interests include biomedical imaging, visual search, and 3D shape recovery.

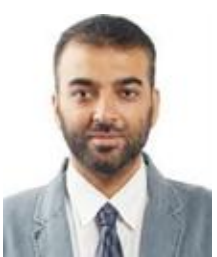

Aamir Saeed Malik received his MSc and Ph.D. from Gwangju Institute of Science \& Technology. South Korea. He is currently Associate Professor and the Director of Mission Oriented Research Biomedical Technology at Universiti Teknologi Petronas. He is senior member of IEEE and member of Australian Pattern Recognition Society. $\mathrm{He}$ is involved in biomedical research since 1999 and has various publications in top-tier journals \& conferences.

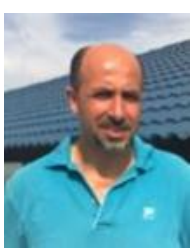

Nidal Kamel received the Ph.D. degree (Hons) from the Technical University of Gdansk, Poland, in 1993. His Ph.D. work was focused on subspace-based array signal processing for direction-of-arrival estimation. Since 1993 he has been involved in research projects related to estimation theory, noise reduction, optimal filtering, and pattern recognition. He developed SNR estimator for antenna diversity combining and introduced the data glove to the area of online signature verification. His present research interest is in brain signal processing for epilepsy assessment and seizure prediction. Currently, he is Associate Professor at the PETRONAS University of Technology. He is IEEE senior member. 